TRANSFER RNA

\section{One State or Many}

from our Molecular Biology Correspondent

THE notion of native and denatured conformations, comparable in terms of base pairing, but differing widely in biological activity, occupies a prominent position in the transfer RNA canon. An adroit attempt to sink a pick in its foundations has nevertheless been made by Biltonen and his colleagues. Their reasoning, based on thermodynamic analysis, is economical and persuasive, and probably wrong withal. The conclusion will not, at all events, much commend itself to workers in the field because of the mass of extant evidence, attesting to the existence of more than one monomeric folded state, but it quite clearly deserves a serious hearing.

In the first place, Levy, Rialdi and Biltonen (Biochemistry, 11, 4138; 1972) have compared equilibrium parameters extracted from optical melting profiles of yeast phenylalanine tRNA with the results of calorimetric measurements. On the assumption that the thermal melting curve represents a two-state process, the fractional change in absorbance at each temperature gives an operational equilibrium constant and free energy of melting, and the van't Hoff relation then leads to a corresponding enthalpy. The latter agrees well with the calorimetric value, thus fulfilling one of the criteria for a two-state process. There are other criteria: the independence of the apparent equilibrium constants of the wavelength of the measurements from which they are derived, in spite of the different sensitivities of different wavelengths to the melting of A-U and of G-C base pairs ; a first-order rate plot for the melting process ; and the absence of discontinuities in the van't Hoff plot. The latter is curved, rather than linear, however, indicating an appreciable heat capacity change on melting (just as is commonly found in the denaturation of globular proteins). The two-state scheme is purely thermodynamic of course, for it does not preclude short-lived intermediates, nor the coexistence of optically indistinguishable substates.

So far, so good-though there is already a head-on conflict with earlier work, reporting multiphasic melting profiles-but the provocative part of the analysis now follows. Levy et al. say that the introduction of magnesium ions displaces the melting curve towards higher temperatures, in the familiar manner, but in no way modifies the twostate scheme. It has been reported further that the enthalpy of interaction of the tRNA with magnesium is essentially zero, and that it is not accompanied by any appreciable absorbance change. The vanishingly low enthalpy is equated with the absence of any con-

formational change on binding of magnesium. On these grounds the authors feel that it is unnecessary to postulate anything more than stabilization of the structured relative to the melted state by preferential binding of magnesium.

Levy and Biltonen develop this view in their second article (ibid., 4145). Using their earlier data for the number of magnesium binding sites in the native and melted states, complete with binding constants for the two classes of sites, and their own values for the thermodynamic parameters of melting, including a heat-capacity term, they can satisfactorily simulate the melting characteristics as a function of magnesium concentration. An interesting consequence of their model is that the entropy of melting, and in consequence also the free energy, change sign at about $16^{\circ} \mathrm{C}$ (in the absence of magnesium), so that low temperature would be predicted to destabilize the structure. They then go on to suggest that the "denatured" state, of low activity, described by Fresco and by Sueoka and their colleagues is nothing more than a mixture of folded and melted molecules, the latter kinetically trapped at the low temperatures by the large activation barriers to refolding. On heating, the barrier is surmounted, and refolding ensues. The magnesium in any case is supposed not to affect the conformation. The burden of proof lies heavy on the authors, however, for they

\title{
Role of Poly I in Interferon Induction
}

THE synthetic polyribonucleotide poly $\mathrm{I}: \mathrm{C}$ is an effective interferon inducer in vivo and in vitro. In investigations to determine what criteria of molecular structure are necessary for interferon induction, it has an advantage over natural RNAs in that the structure of its strands may be altered and the effects of compounds of varying molecular weights can be found.

During the past two years there have been several conflicting reports on whether the respective sizes of the poly I and poly $\mathrm{C}$ moieties affect the biological activity of poly I: C. Probably one of the difficulties in this field is that neither the same systems nor the same methods for molecular weight determinations are used. Two recent reports, however, indicate that it is the size and intactness of the poly I strand which are important for the biological activity of the complex.

Mohr, Brown and Coffey, in next Wednesday's Nature New Biology (December 20), report their investigations on the relation between the size of poly I when complexed to poly $\mathrm{C}$ for the induction of interferon and also for affecting the survival time of mice injected with leukaemia cells. They found that it was the size of the poly I have to shrug off fractionations of native and denatured forms of tRNAs, on columns for example, one component being active, the other scarcely so, even though their optical properties differ little ; also the different nuclease digestion patterns, which are not readily explained in terms of mixtures of states. The most explicit results on these lines have recently been described by Streeck and Zachau (Europ. J. Biochem., 30, 382 ; 1972). The argument may ultimately hinge on how different two states may be conformationally, and yet be thermodynamically equivalent within reasonable experimental error. If, as Fresco and his colleages suggest, the native and denatured states do not differ widely in base pairing, they could well be near-degenerate in enthalpic terms.

Just failing to make direct contact with Levy and Biltonen, because the ionic conditions are not directly comparable, and the tRNA is not the same species, is a group of three articles by Crothers et al. What they find, however (Cole, Yang and Crothers, Biochemistry, 11, 4358; 1972), is that several purified $t R N A s$ give multiphasic melting curves at high salt concentrations. In terms of transitions that depend on ionic strength and temperature, phase maps can be compiled. These indicate that the molecules can exist in four conformational states, one of which, in the high-salt and low-temperature range, is the native molecule, and another the

moiety and not the poly $\mathrm{C}$ moiety which was the crucial factor. Poly I affects the stability of the complex to a much greater extent than poly $\mathrm{C}$ : a decrease in the size of poly I decreased the $T_{m}$ of the complex significantly, whereas a similar decrease in the size of poly $\mathrm{C}$ had very little effect. These results are in close agreement with those obtained by DeClerq and DeSomer ( $J$. Virol., 9, 721 ; 1971).

Carter et al. (J. Mol. Biol., 70, 567 ; 1972) used poly $I: C$, in which one of the strands was interrupted by unpaired bases for interferon induction, and found that if the $\mathrm{C}$ strand was affected the complex was still active, whereas if the I strand was affected there was very little activity. The $T_{m}$ 's of these modified complexes were well above the incubation temperatures of the experiments, and protection of the complexes from nucleases using polylysine did not increase the activity so that it seems that neither thermal stability nor nuclease resistance are the only criteria involved in interferon induction, as has sometimes been suggested. Further investigations on the importance of the poly I moiety of poly I : C may help to elucidate the mechanism of interferon induction. 